\title{
LIGA DE DOR: UMA EXPERIÊNCIA DE ENSINO EXTRACURRICULAR
}

\author{
LEAGUE AGAINST PAIN: \\ AN UNDERGRADUATE EXTRACURRICULAR PROGRAM
}

\author{
Cibele Andrucioli de Mattos Pimenta * \\ Manoel Jacobsen Teixeira ** \\ Patrícia Simões *** \\ César Simões **** \\ Diná de A. Lopes Monteiro da Cruz ${ }^{\star * * * *}$

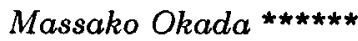

PIMENTA, C.A. de M. Liga de dor: uma experiéncia de ensino extracurricular. Rev.Esc.Enf.USP, v.32, n.3, p. 281-9, out. 1998.

\begin{abstract}
RESUMO
Os objetivos deste estudo foram descrever um programa extracurricular de educação em dor, organizado na forma de Liga, e identificar a opinião dos alunos sobre esse modelo de ensino. O Estatuto e o Reginento Interno da Liga de Dor foram as fontes de dados para a descrição da sua organização e funcionamento. Todos os alunos que frequentaram a Liga de Dor nos anos de 1995 e 1996 foram solicitados a responder um questionário sobre suas experiências na Liga. A Liga de Dor foi organizada sob o patrocínio do Centro Acadêmico Osvaldo Cruz, da Faculdade de Medicina da USP, e Centro Acadêmico. XXXI de Outubro, da Escola de Enfermagem da USP, em 1995. É composta por alunos dessas duas faculdades e profissionais enfermeiros e médicos, de várias especialidades. Todas as atividades são voluntárias, desenvolvidas em período extracurricular e os estudantes dirigem a Liga. Os objetivos da Liga são: melhorar a qualidade do ensino sobre dor nas escolas médica e de enfermagem, desenvolver pesquisas relativas à epidemiologia, clínica e controle da dor e promover modelo de assistência multidisciplinar e multiprofissional. A maioria dos alunos expressou ter alcançado seus objetivos, total ou parcialmente; ter aumentado seu conhecimento sobre as sindromes álgicas e sobre o manejo do doente com dor; sentir-se gratificado com sua atividade; que o tema dor deveria ser incluído no currículo de modo mais específico e que recomendaria a outros colegas a participação na Liga de Dor. As opiniões dos estudantes mostraram que a Liga de Dor pode ser um modelo útil para a introdução do ensino da dor nos cursos de graduação de enfermagem e de medicina.
\end{abstract}

UNITERMOS: Dor. Dor crônica. Educação em dor. Educação em enfermagem. Educação médica.

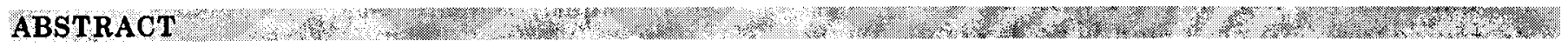

Although anatomical and physiological base of pain are subject of the basic sciences in health undergraduate curriculum, it is not usually to have pain concepts and therapy into undergraduate programs. Describing the League Against Pain objectives and organization and the students'opinions about their participation in this league were the aim of this stduy. The League Against Pain Statute was consulted to describe its objectives and organization. The opinions of all of the students that attended the League Against Pain program in 1995 and 1996 were investigated. The League Against Pain was organized at an university hospital in 1995. It is composed by nurses and medical students and professionals (physicians in varied specialties and nurses). All the activities are voluntary and the students are the managers of the League. The ovjectives of the League are: improving the quality of teaching of pain subjects in nursing and medical schools; developing research in epidemyological, clinical and therapeuthics aspects

* Prof Dra do Depto de Enfermagem Médico-Cirúrgica da Escola de Enfermagem da Universidade Sao Paulo.Coordenadora de Enfermagem da Liga de Dor.

** Prof. Dr. do Departamento de Neurologia cla Faculdade de Medicina da Universidade de Sao Paulo. Coordenador Geral da Liga de Dor.

... Aluna de graduaçáo da Escola de Enfermagem da Universidade de São Paulo. Membro da Liga de Dor.

**** Aluno de graduação da Faculdade de Medicina da Universidade de São Paulo. Membro da Liga de Dor.

**** Prof Dræ do Depto de Enfermagem Médico-Cirúrgica da Escola de Enfermagem da Universidade São Paulo. Orientadora da Liga de Dor.

*n*** Neurologista. Médica cla Divisão de Clínica Neurológica do Hospital das Clínicas da FM-USP. Coordenadora Médica da Liga de Dor. 
of pain and to promote a model of multidisciplinary and multiprofessional assistance. The most frequent students' opinions about their participation in the League were: their habilities in pain control and in professional and client relationship were improved; they achievied their objectives; that pain should be included into undergraduate courses; and they would recommend the League for other students. The results are promising. The students' opinions about their experience in the League Against Pain have showed that the League Against Pain could be an usefull model to introduce pain subjects to undergraduate nursing and medical students.

UNITERMS: Pain. Chronic pain. Education on pain.

\section{INTRODUÇÃO}

A literatura biomédica cresce $6 \%$ a $7 \%$ a cada ano, dobra a cada $10-15$ anos e aumenta 10 vezes a cada 35-50 anos. Alguns temas como os relacionados a biologia molecular e imunologia expandem-se com mais velocidade ${ }^{21}$. O sistema de assistência está também sofrenclo grandes modificações. Diagnósticos precoces, aplicação de terapias eficazes nas áreas relacionadas ao controle da doença com resultados favoráveis são exigências do moderno aparelho assistencial. Paralelamente, observa-se grande progresso nas áreas relacionadas ao controle dos sintomas do que resulta a necessidade de aumentar e melhorar a transmissão dos conhecimentos e a compreensão dos cuidados destinados aos doentes. Entretanto, a melhora dos programas educacionais comunitários e especialmente na área da saúde não ocorrem com a mesma velocidade com que os conhecimentos se multiplicam.

Muitas escolas incluem em seus currículos matérias descritivas em detrimento dos temas analíticos e que envolvem pensamento crítico e logico. A dor inclui-se en tre esses últimos. É através dela que a maioria das afecções manifesta-se. Como sintoma ou doença é frequentemente objeto da procura pelo sistema de saúde. De sua análise crítica é que, independentemente do procedimento complementar, o diagnóstico é estabelecido e as estratégias terapêuticas visando ao seu controle ou à eliminação da situação causal são propostas. $O$ insatisfatório controle da dor aguda e crônica está, exaustivamente, registrado em estudos realizados em países do hemisfério norte e em alguns estudos nacionais ${ }^{2,6,15.23,24,25,34,35}$. Entretanto, o tema dor é geralmente negligenciado pelos educadores encarregados da elaboração dos currículos escolares, mesmo nas áreas em que a dor é muito prevalente, como a oncologia ${ }^{28}$. Também os livros de texto versando sobre assuntos em que a dor é muito frequente, como os que enfocam patologias traumáticas dedicam pouca atenção ao assunto $0^{40}$. A necessidade da inclusão especializada do tema em cursos de graduação, aperfeiçoamento, possgraduação e de reciclagem e maior número de publicações acessíveis foi atestada em inquérito recentemente publicado envolvendo profissionais de saúde em nosso meio ${ }^{35}$. Medidas educativas e de pesquisa para esclarecer a importância da dor como sintoma e como doença e que enfatizem a necessidade de avaliação e controle rigorosos, são ainda necessárias.

Embora as bases anatômicas e fisiológicas da sensibilidade seja assunto das ciências básicas das disciplinas da área de saúde, conceitos e terapias sobre dor não são, tradicionalmente, incluídos nos currículos de graduação. A criação de novas disciplinas é muito complexa, pois é necessário modificar a carga horária e a distribuição dos temas e a organização de currículos com disciplinas optativas, ainda é pouco frequente nas escolas tradicionais. Além disso, a introdução de novos tópicos, usualmente, requer longo tempo de maturação após o desenvolvimento dos conceitos e das técnicas terapêuticas. Frente à necessidade de se aprimorar a educação em dor, nos aspectos clínicos e de pesquisa, para alunos de graduação em medicina e em enfermagem, foi organizada a Liga de Dor do Centro Acadêmico Osvaldo Cruz, da Faculdade de Medicina da USP e do Centro Acadêmico XXXI de Outubro, da Escola de Enfermagem da USP, em março de 1995. A Liga de Dor está vinculada à Divisão de Clínica Neurológica do Hospital das Clínicas da Faculdade de Medicina da Universidade de São Paulo.

Os objetivos deste estudo foram descrever um programa extracurricular de educação em dor, organizado no modelo de Liga, e identificar a opinião dos alunos sobre esse modelo de ensino.

\section{MÉTODO}

Para a descrição da estrutura e funcionamento da Liga de Dor utilizou-se como fonte de dados 0 Estatuto e o Regimento Interno da Liga de Dor.

As opiniões dos estudantes sobre o treinamento que receberam foram identificadas por questionário, sem identificação do respondente, elaborado especialmente para esse fim e previamente testado quanto a sua adequação aos objetivos deste estudo. Os dados foram colhidos no segundo semestre de 1996 e todos os estudantes de enfermagem (12) e medicina (22) que frequentaram 
a Liga de Dor nos anos de 1995 e 1996 foram convidados a participar. Os respondentes perfizeram $100 \%$ (12) das alunas de enfermagem e 86,4\% (19) dos estudantes de medicina.

\section{RESULTADOS}

A Liga de Dor é regida por Estatuto, registrado em cartório, que prevê suas finaliclades, composição e órgãos dirigentes. O Regimento Interno regula a administração, funcionamento e atribuições dos integrantes. Todas as atividades são voluntárias e realizadas em período extracurricular.

A Liga de Dor tem finalidades assistenciais, de ensino e pesquisa, relacionadas ao tema dor. As possíveis categorias de membros são: aspirante, efetivo, coordenador, orientador, colaborador, pesquisador, estagiário e visitante. Os acadêmicos fazem parte das 2 primeiras categorias. O membro aspirante torna-se efetivo ao completar $75 \%$ de frequência na Liga por seis meses consecutivos. Alunos, na conclição de membros efetivos, das duas instituições compõem a Diretoria da Liga de Dor e respondem pela gerência das atividades. São orientados por professores da Escola de Enfermagem da USP e da Faculdade de Medicina da USP e por Médicos Assistentes do Complexo HC-FMUSP.

A Liga possui Coordenador Geral e Coordenadores Médico e de Enfermagem. Dentre as especialidades médicas conta com neurologista, ortopedista, anestesista, psiquiatra, fisiatra e neurocirurgião.

Anualmente é feito Curso Introdutório à Liga de Dor com 15 horas de duração onde são desenvolvidos temas relacionados a fisiopatologia da dor, avaliação da experiência dolorosa, síndromes dolorosas mais frequentes, bases conceituais das terapias analgésicas farmacológica, fisioterápica e neurocirúrgica e sobre a atuação dos profissionais de enfermagem e da saúde mental na Liga de Dor. Os alunos que frequentam o curso e desejam ser membros da Liga são submetidos a teste de seleção. Os classificados frequentam a Liga de Dor por 11 meses, durante 3 horas por semana. Os estudantes desenvolvem, sob a orientação dos profissionais, atividades de assistência aos doentes (consulta e realização de alguns procedimentos) e participam no desenvolvimento de projetos de pesquisa. Participam, também, de aulas e discussões de casos.

As opiniões dos estudantes sobre o programa do qual participaram podem ser observadas nas tabelas e nos relatos que se seguem.

Tabela 1 - Freqüência das respostas dos alunos quanto aos motivos que os levaram a participar da Liga de Dor. São Paulo, 1996.

Alunas $\quad$ Alunos Total

Motivos

Enfermagem

Medicina

\begin{tabular}{lcrrrrr} 
& $\mathbf{n}^{\circ}$ & \multicolumn{1}{c}{$\%$} & $\mathbf{n}^{\circ}$ & $\%$ & $\mathbf{n}^{\circ}$ & $\%$ \\
\hline Abrangência e interesse pelo tema & 8 & 36,4 & 16 & 40 & 24 & 38,7 \\
Aperfeiçoar conhecimento sobre dor & 6 & 27,3 & 4 & 10 & 10 & 16,1 \\
Desenvolver trabalhos científicos & 2 & 9,1 & 2 & 5 & 4 & 6,4 \\
Aprender consulta e vinculo profissional/paciente & 4 & 18,2 & 5 & 12,5 & 9 & 14,5 \\
Desejo de ajuclar pessoas com dor & 2 & 9,1 & 1 & 2,5 & 3 & 4,8 \\
Por ser multidjsciplinar & - & - & 1 & 2,5 & 1 & 1,6 \\
Interesse por neurologia/neurocirurgia & - & - & 11 & 27,5 & 11 & 17,7 \\
TO'TAL & 22 & 100,0 & 40 & 100,0 & 62 & 100,0 \\
\hline
\end{tabular}

Na Tabela 1 porle-se observar que as razões mais apontadas para participar da Liga de Dor foram a abrangência e interesse pelo tema dor, aperfeiçoar o conhecimento na área e, para os alunos de medicina, o interesse por neurologia e neurocirurgia. Cadla aluno expressou, em méclia, 2 razões para participar da liga de Dor.

Indagados se, decorrente de suas atividades na Liga de Dor, houve melhora do seu conhecimento e habilidade para lidar com o doente com dor, todos os alunos (100,0\%) responderam positivamente e enfatizaram a articulação teórico-prática como o aspecto fundamental que possibilitou a melhora no desempenho. Ainda, todas as alunas de enfermagem e a maioria $(78,9 \%)$ dos estudantes de medicina expressaram que a Liga facilitou/promoveu o trabalho em equipe.

Incuiridos sobre a participação em projetos de pesquisa 2 alunas de enfermagem (16,6\%) e 9 alunos de medicina $(47,4 \%)$ responderam não ter participado. Todas as alunas de enfermagem e 78,9\% dos alunos de medicina relataram que a participação na Liga os motivou a frequentar encontros cientificos sobre dor. 
A Tabela 2 mostra a frequência de respostas dos alunos quanto ao alcance ou não dos objetivos iniciais da participação na Liga de Dor. Observa-se que, no conjunto dos alunos, a frequência dos que responderam que seus objetivos foram alcançados totalmente foi semelhante a dos alunos que responderam que foram alcançados parcialmente.

Tabela 2 - Freqüência de respostas segundo o alcance dos objetivos desejados junto à Liga de Dor. São Paulo, 1996.

\begin{tabular}{|c|c|c|c|c|c|c|}
\hline \multirow{2}{*}{$\begin{array}{c}\text { Alcance dos } \\
\text { objetivos }\end{array}$} & \multirow{2}{*}{$\begin{array}{c}\text { Alunas } \\
n^{\circ}\end{array}$} & \multirow{2}{*}{$\begin{array}{c}\text { Enfermagem } \\
\%\end{array}$} & \multirow{2}{*}{$\begin{array}{c}\text { Alunos } \\
n^{\circ}\end{array}$} & \multirow{2}{*}{$\begin{array}{c}\text { Medicina } \\
\%\end{array}$} & \multicolumn{2}{|c|}{ Total } \\
\hline & & & & & $n^{\circ}$ & $\%$ \\
\hline Sim & 10 & 83,3 & 6 & 31,6 & 16 & 51,6 \\
\hline Em parte & 2 & 16,7 & 12 & 63,2 & 14 & 45,2 \\
\hline Não & - & . & 1 & 5,2 & 1 & 3,2 \\
\hline TO'TAL & 12 & 100,0 & 19 & 100,0 & 31 & 100,0 \\
\hline
\end{tabular}

As razões que os alunos apontaram para o podem ser examinadas na Tabela 3. alcance parcial dos seus objetivos foram diversas e

Tabela 3 - Freqüência das respostas dos alunos quanto às razões do alcance parcial dos objetivos desejados junto à Liga de Dor. São Paulo, 1996.

\begin{tabular}{|c|c|c|c|c|c|c|}
\hline \multirow{3}{*}{ Razões } & \multicolumn{2}{|c|}{ Alunas } & \multicolumn{2}{|c|}{ Alunos } & \multicolumn{2}{|c|}{ Total } \\
\hline & \multicolumn{2}{|c|}{ Enfermagem } & \multicolumn{2}{|c|}{ Medicina } & \multirow[b]{2}{*}{$\mathrm{n}^{\circ}$} & \multirow[b]{2}{*}{$\%$} \\
\hline & $\mathrm{n}^{\circ}$ & $\%$ & $\mathrm{n}^{\mathrm{o}}$ & $\%$ & & \\
\hline Não participação em projetos de pesquisa & 1 & 50 & 2 & 15,4 & 3 & 20 \\
\hline Pouca execução de procedimentos específicos & 1 & 50 & 1 & 7,7 & 2 & 13,3 \\
\hline Poucas aulas & $\cdot$ & - & 2 & 15,4 & 2 & 13,3 \\
\hline Tempo nas discussões de caso poderia ser menor & - & - & 4 & 30,7 & 4 & 26,7 \\
\hline Pouco tempo para o atendimento & $\cdot$ & $\cdot$ & 2 & 15,4 & 2 & 13,3 \\
\hline Insatisfação com o tratamento ofereciclo & $\cdot$ & - & 1 & 7,7 & 1 & 6,7 \\
\hline Limitação pessoal: pouco tempo & $\cdot$ & - & 1 & 7,7 & 1 & 6,7 \\
\hline TOTAL & 2 & 100,0 & 13 & 100,0 & 15 & 100,0 \\
\hline
\end{tabular}

Quando indagados, todos os alunos (100,0\%), responderam ter experimentado momentos de gratificação advindos de suas atividades na Liga de
Dor e apontaram os mais relevantes, conforme se pode observar na Tabela 4.

Tabela 4 - Freqüência das respostas dos alunos quanto às razões para o sentimento de gratificação com suas atividades na Liga de Dor. São Paulo, 1996.

\begin{tabular}{lccrrrrr} 
& \multicolumn{2}{c}{ Alunas } & \multicolumn{2}{c}{ Alunos } & \multicolumn{2}{c}{ Total } \\
\multicolumn{1}{c}{ Razões } & \multicolumn{2}{c}{ Enfermagem } & \multicolumn{2}{c}{ Medicina } & \\
& \multicolumn{1}{c}{$\mathrm{n}^{\circ}$} & $\%$ & $\mathrm{n}^{\circ}$ & $\%$ & $\mathrm{n}^{\circ}$ & $\%$ \\
\hline Reconhecimento do doente & 6 & 37,5 & 4 & 14,8 & 10 & 23,3 \\
Participação em projetos de pesquisa & 4 & 25,0 & - & - & 4 & 9,3 \\
Experiência/conhecimento adquirido & 3 & 18,7 & 5 & 18,5 & 8 & 18,6 \\
Melhora do doente & 3 & 18,7 & 17 & 63,0 & 20 & 46,5 \\
Trabalho multiprofissional & - & - & 1 & 3,7 & 1 & 2,3 \\
TOTAL & 16 & 100,0 & 27 & 100,0 & 43 & 100,0 \\
\hline
\end{tabular}


Quanclo inquiridos se o que lhes foi ensinado nos cursos de graduação é suficiente para cuidar de doentes com dor observou-se que pequena parcela respondeu sim (2 estudantes de enfermagem e 1 de medicina) e o restante distribui-se, de modo equitativo, entre as alternativas "não" e "em parte". Todas as alunas de enfermagem e $18(94,7 \%)$ alunos de medicina julgam que esse tema deve ser incluído, de modo mais enfático, nos cursos de graduação. Finalizando a avaliação indagou-se aos alunos se recomendariam a um colega participar da Liga de Dor. Todlas as estudantes de enfermagem e 17 $(89,4 \%)$ dos de medicina disseram sim.

\section{DISCUSSÃO}

O controle da dor é atribuição de todo profissional de saúde. Conceitos fundamentais a respeito dos mecanismos de dor e de suas repercussões nas áreas física, emocional e social dos indivíduos, das possibilidades diagnósticas e sobre os princípios da terapêutica, devem ser conhecidos por todos aqueles que atuam em qualquer campo das ciências da saúde. A maioria dos alunos deste estudo (90.3\%) não considerou suficiente o que aprendeu sobre dor nos cursos de graduação.

As escolas de medicina em especial, e as de saúde em geral, têm a graduação fundamentada no ensino com visão biocêntrica/tecnocêntrica ${ }^{14}$. $O$ corpo humano é estudado por partes e a doença vista como mal funcionamento dos mecanismos biologicos. O papel do médico é o de intervir física ou quimicamente para normalizar ofuncionamento da unidade comprometida. A finalidade principal parece ser a de formar estudiosos em doenças, principalmente especialistas atuando em hospitais, e não de capacitar pessoal para cuidar de pessoas doentes. Tal modelo resulta em insuficiente visão do indivíduo como um todo e do inclivíduo dentro da sociedlade. A preocupação sobre a relação entre condições biologicas, psicológicas, sociais, culturais e a expressão e solução de questões de saúde teve como marco importante a reunião de Alma Ata em 1978 e implicou na recomendação de que esses conceitos fossem incluídos na educação básica. Almeja-se formar profissionais capazes de contribuir para o bem-estar físico, psíquico e social dos doentes ${ }^{3}$. O currículo deve ter um conteúdo geral e básico comum complementado por temas que contemplem as especificidades epidemiologicas e as necessidades de saúde regionais ?

Dor é um fenômeno complexo, multifatorial e subjetivo. onde aspectos biológicos, sócio-culturais e emocionais estão envolvidos. A formação dos profissionais de saúde para a avaliação e manejo da dor deve contemplar esta abrangência.
Tem sido recomendada a inserção no curso de graduação dos conteúdos pertinentes ao tema dor distribuídos entre as disciplinas das áreas básica, as de conteúdo clínico, naquelas relativas ao sistema de saúde e na disciplina sobre metodologia de pesquisa ${ }^{8,39}$. A complexidade da dor e de suas repercussões requerem a integração de conhecimentos comportamentais e biológicos em ambiente em que professores de ciências básicas e de ciências aplicadas de várias disciplinas possam interagir, promovendo entusiasmo real sobre as questões da dor.

Em escolas que acompanham o modelo de formação clássico, a fỉm de não comprometer a estrutura global do ensino, os temas sobre clínica e terapêutica da dor podem integrar-se a alguma disciplina da área médica ou cirúrgica, preferencialmente de ambas. Em escolas com currículo nuclear pode constituir disciplina especifica ${ }^{27}$. Na Faculdade de Medicina da Universidade e São Paulo e na Escola de Enfermagem da Universidade de São Paulo, a solução para a complementação educacional foi a criação da Liga de Dor. Havia experiência anterior com a Liga de Cefaléia e de Algias Craniofaciais ${ }^{32}$

A proposta do modelo de funcionamento e organização da Liga da Dor foi elaborada a partir de concepções de seus organizadores e fundamentam-se nos princípios:

-dor é uma experiência onde aspectos biológicos, emocionais e culturais estão ligados de modo indivisível. O ensino sobre dor deve prover informação para que estes aspectos possam ser adequadamente considerados, investigados e manejados;

-as intervenções terapêuticas devem, sempre que possivel, atuar na "causa" da dor. Terapias que pouco intervenham na fisiologia e no comportamento normal, pouco complexas, menos dispendiosas e com pequeno potencial de complicações e efeitos adversos são desejáveis;

- o conceito de qualidade de vida e o respeito aos princípios éticos em saúde, devem permear toda a atividade de ensino, pesquisa e assistência, incluindo o processo de tomada de decisão terapêutica;

-a experiência assistencial é a possibilidade de integração dos conceitos que envolvem dor e seu manejo. O treinamento em dor deve incluir o atendimento a doentes por todos os profissionais de saúdle.

A Associação Internacional para o Estudo da Dor (IASP) propôs temas em educação sobre dor para os profissionais de saúde, visando ser referência para a elaboração de programas de treinamento e para a previsão de recursos humanos e materiais necessários na organização de clínicas de dor. O 
programa curricular foi elaborado por comitê de especialistas em 1991 e revisto em $1995^{10}$. Consiste de 29 capítulos. Em cada capítulo estão apontados, de forma sumária, os componentes básicos do tema, o que está, ou não, bem estabelecido na literatura e as referências bibliográficas. Os capítulos referentes a fisiopatologia da dor compreendem temas de anatomia, fisiologia e farmacologia envolvidos na transmissão e modulação da dor. A avaliação e mensuração da dor em humanos, a identificação e atribuição de significado às diferentes qualidades da dor e a compreensão dos aspectos psicossociais envolvidos na experiência dolorosa são também enfocados. A proposta educacional no que se refere às terapias antiálgicas compreencle o estudo dos analgésicos opiáceos, dos antiinflamatórios não hormonais, dos antidepressivos, anticonvulsivantes e corticosteróides, entre outros. Compreende também temas sobre medicina física e procedimentos cirúrgicos neuro-ablativos ou destinados à estimulação do sistema supressor de dor. Bloqueios do sistema nervoso periférico, terapias psicologicas e cognitivo-comportamentais são também incluídas entre as alternativas terapêuticas para o controle da dor. O estudo das principais síndromes álgicas (lombalgia, dor miofascial, dor neuropática, cefaléia, dor orofacial e (lores reumatológicas) compõe a recomendação para o treinamento em dor da IASP. Há também capítulos dedicados à dor no doente com câncer, no pós-operatório e na criança. Não há, entretanto, no programa educacional, recomendações específicas sobre o controle da dor no idoso e no doente com infecção pelo vírus HLTV. 2/SIDA. Há, ainda, capítulos com considerações sobre a atuação de equipes multidisciplinares e multiprofissionais em dor aguda e sobre desenhos de pesquisa em dor. Finalizando, os aspectos éticos na investigação e no tratamento da dor em seres humanos e em animais, são também discutidos.

A educação para o controle da dor de origem neoplásica vem sendo enfatizada na Liga de Dor, visto a alta frequência de ocorrência de cânceres na população, do sintoma doloroso que frequentemente a acompanha co insuficiente controle que tem sido descrito na literatura dos países de primeiro mundo ${ }^{2,5.6,9} \mathrm{e} \mathrm{cm}$ nosso meio ${ }^{23}$. Os esquemas básicos para controle da dor no doente oncológico divulgados através do Programa para Controle da Dor no Câncer da Organização Mundial de Saúde ${ }^{22}$, devem nortear as ações desenvolvidas, o que vem sendo feito na Liga de Dor.

Diversas são as razões do não controle da dor aguda e crônica. A falta de informações na comunidade e de informações e treinamento entre os profissionais encarregados da assistência à saúde são apontadas como as principais entre as múltiplas causas do insatisfatorio alívio das queixas álgicas, especialmente as crônicas. A avaliação imprecisa do quadro álgico, nos aspectos de etiologia e características; falhas na formação dos profissionais quanto ao conhecimento sobre os métodos e fármacos analgésicos adequados para o alívio da dor; a crença popular de que algumas síndromes álgicas, por exemplo as relacionadas ao câncer são inevitáveis e incontroláveis; o medo exagerado do desenvolvimento de dependência psicológica com o uso de opiáceos para o controle da dor aguda intensa ou cla clor de origem neoplásica e a subestimação dos aspectos afetivos envolvidos na experiência dolorosa, principalmente os quadros depressivos, são alguns fatores que podem ser destacados $11,15,16,23,24,26,29,30,35$.

Os programas educacionais devem apresentar quatro características: identificação das necessidades educacionais de grupos específicos, estabelecimento de objetivos claros, uso de métodos que possibilitem a participação ativa dos estudantes e avaliações para permitir reajustamentos da programação e da metodologia de ensino ${ }^{19}$. Modelos teóricos sem exemplificação prática resultam em pequena retenção de informações pelos alunos. $O$ melhor método é o que mistura a participação ativa com a apresentação de experiências educacionais que reforcem fatos e auxiliem os estudantes a adotar atitudes positivas frente a dor, com conceitos tebricos sobre tratamento e avaliação global do indivíduo em sofrimento ${ }^{40}$. As atividades que vêm sendo realizadas na Liga de Dor apresentam estas características, o que foi atestado pelos alunos deste estudo que relataram, em $100 \%$ das vezes, que a Liga de Dor lhes permitiu aperfeiçoar o conhecimento sobre o tema e enfatizaram a importância da articulação teoria e prática clínica para a obtenção deste resultado.

Há evidências de que a introdução precoce de temas abrangentes no curso de graduação reduz as atitudes negativas durante os anos em que temas visando a formação clínica são ministrados ${ }^{10}$. Wilson et al ${ }^{40}$ organizaram curso de 6 horas durante duas semanas versando sobre introdução em conceitos básicos sobre dor, contato com doentes com dor e acompanhamento de médicos atendendo doentes com dor. $Q$ curso constou de exposições sobre teorias e conceitos sobre dor, tratamento da dor aguda, tratamentos comportamentais da dor, dor no câncer e dor na infância. Observação do atendimento multidisciplinar do doente com dor crônica, da execução de técnicas de tratamento e discussões em grupo sobre situações clínicas e sobre temas de dor complementaram o curso. Observaram, por meio de inquérito realizado cinco meses apos o término do curso, que o conhecimento sobre a prevalência da dor em algumas condições agudas e crônicas e sobre a real participação de condições psíquicas na origem 
e expressão da dor, mostrou-se expressivamente melhor. Os alunos tornaram-se motivados a dar mais atenção às condições dolorosas, passaram a considerar esta questão mais importante que antes do curso e perceberam que a atuação do profissional no tratamento da dor é gratificante. Os conceitos, principalmente os relacionados com assuntos clinicamente relevantes, mais do que aqueles teoricamente relevantes foram retidos por significante número de estudantes.

A versão de temas teóricos em modelos de ação clínica, torna o aprendizado mais satisfatório ${ }^{38}$. O modelo de ensino proposto na Liga de Dor pelas características de extensão (11 meses), periodicidade (semanal), articulação teórico-prática (aulas, atendimento aos doentes, discussão dos casos e desenvolvimento de pesquisas) parece ser modelo que propicia a retenção de informações e adequação da postura frente à dor. A satisfação dos alunos com a participação na Liga de Dor pode ser inferida pois a maioria dos alunos $(93,1 \%)$ recomendaria a um colega participar dessa atividade e $93,5 \%$ referiu que seus objetivos estavam senclo atendidos total ou parcialmente.

A educação deve também ser estendida para a comunidade. Doentes estóicos ou que admitem ser a dor e o sofrimento condições que necessariamente devem ser suportadas, mais que controladas, necessitam ser educados. Necessitam ser informados sobre possibilidades de tratamento da dor e de como interagir com as equipes de saúde ${ }^{13}$. Doentes melhor orientados solicitam mais atenção para o controle de seu desconforto e determinam melhora clas condições de assistência ${ }^{4}$.

No Brasil há algumas experiências sobre o ensino de dor para alunos de graduação, pós. graduação sensu-strictu e em programas de aperfeiçoamento. Faltam registros na literatura sobre estas experiências. Há necessidade de proposta nacional sobre educação em dor para os diversos profissionais da saúde, nos diferentes âmbitos de formação. É também premente a organização de estratégias para a divulgação e educação sobre o tema junto aos profissionais e leigos.

Para modificar e melhorar a educação técnica e muclar as atitudes que refletem as tradições filosoficas não basta apenas informar. Apenas a comunicação de idéias não modifica 0 comportamento, que é especialmente determinado por padrões estabelecidos pela prática e pelas tradições ${ }^{16}$. Para muitos profissionais de saúde há predomínio da visão biológica sobre dor, embora não haja modelo teórico que responda a todos os questionamentos que envolvem dor, sua expressão e controle ${ }^{18.34}$. Há aspectos, além dos biológicos, como os culturais e afetivos, envolvidos na vivência e expressão da dor que são, freqüentemente. negligenciados. Dor é um conceito que culturalmente variou com a história. Aristoteles a considerava uma emoção e Descartes como uma sensação. Atualmente, dor é considerada uma sensação associada a importante expressão emocional com significado simbólico negativo $0^{10,18,34}$. Os significados atribuílos à dor e aos métodos para seu controle estão relacionados às características do indivíduo e aos valores e crenças culturais e cientificas de cada epoca ${ }^{18}$. O treinamento dos profissionais na eluciclação da etiologia da dor, na identificação de fatores sócio-psico-culturais que podem estar contribuindo para seu agravamento ou manutenção e nos métodos para seu controle deve compor o programa de educação em dor.

Ansiedade, hostilidade e depressão são as respostas emocionais mais estudadas na experiência dolorosa. Depressão é freqüente na dor crônica, variando entre entre $22 \%$ e $78 \%$ de acordo com uns ${ }^{1,30}$ e entre $10 \%$ a $30 \%$ de acordo com outros ${ }^{17}$. A associação dor e depressão pode agravar, de modo acentuado, a funcionalidade e sofrimento do doente. Depressão pode exarcebar a magnitude do quadro álgico, comprometer a adesão ao tratamento e a resposta aos analgésicos, acarretar maior isolamento social, desesperança e privação de cuidados ${ }^{1,17,23,36}$.

Há também aspectos éticos envolvidos no manejo da dor, que devem ser considerados na atividade clínica diária e discutidos com os alunos. Quatro princípios cardinais compõem a base da ética médica: autonomia, beneficência, não maleficência e justiça. Autonomia é o direito de outrem de agir de acordo com seus próprios valores e crenças. E o oposto do paternalismo: "eu sei o que é melhor para você". Doente e profissionais compartilham as decisões e a responsabilidade sobre elas. Beneficência representa promover o bem estar, previnir ou remover danos. O princípio da nãomaleficência visa à não infligir mal, causar dano ou injúria. É o contrapeso para o princípio de beneficência e ambos estão profundamente imbricados com a autonomia do doente ${ }^{12,37}$. O princípio de justiça representa a imparcialidade, o direito de todos acessarem aos serviços, profissionais de saúde e recursos terapêuticos. O uso de tecnologia dispendiosa para o controle da dor, quando métodos mais simples e econômicos são possíveis, fere o princípio de justiça, considerando-se que nem todos conseguem acesso a ela e a distribuição dos recursos econômicos de forma eqüitativa é impraticável ${ }^{12,37}$. Embora não incluídos entre os cardinais, os princípios de efeito duplo, integridade dos profissionais de sáude, fidelidade e veracidade compõem o escopo para a análise de situação e tomada de decisões em saúde. Efeito duplo possibilita discriminar a intenção primária da ação do efeito indesejavel que pode acompanhá-la. Uma 
terapêutica que visa primariamente trazer conforto como, por exemplo o uso de opiáceos para o controle da dor oncológica, pode ser acompanhada de efeitos indesejáveis como depressão respiratória mas, é eticamente válida, pela real intenção da ação. Ainda, os profissionais de saúde têm obrigação de se manter fiéis, de não abandonar os doentes, de cumprir as promessas expressas ou subentendidas, de ser sinceros, de não enganar as pessoas e o direito de, respeitados os princípios éticos, conservar sua opção religiosa e crenças morais ${ }^{12}$.

A avaliação dla real condição de um doente com dor e dos resultados do tratamento, não depende apenas do dimensionamento de um dos componentes da expressão dolorosa, o sensitivo. A aferição dos aspectos de comportamento, psíquico e das repercussões sociais, profissionais, econômicas, as modificações da fisiologia de diferentes sistemas e aparelhos, a necessidlade do uso de adlaptações para o desempenho de ativiclades de vida prática e diária, devem ser aferidas. O objetivo do tratamento é a melhora da qualidade de vidla e não apenas da dor, isoladamente. Qualidade de vida é conceito subjetivo e pode ser compreendido como a sensação íntima de conforto, bem estar ou felicidade no desempenho de atividades físicas, intelectuais e psíquicas, dentro da realidade pessoal, familiar e dos valores da comunidade em que o inclivícluo está inserido ${ }^{20}$. A importância desse conceito reside no fato de possibilitar ao profissional retirar o foco de atenção da doença/dor para centrá-lo no indivíluo. O alívio da dor favorece a melhora da qualidade de vida em significativa parcela dos doentes.

Na Liga de Dor, estudantes de enfermagem e medicina seguem roteiro de entrevista semiestruturada para a realização das consultas iniciais e de retorno dos doentes. Esses roteiros, elaborados especificamente para este fim e para cada um dos grupos de alunos, possuem abrangência que permite a avaliação do doente nos âmbitos biologico emocional e sócio-cultural. Os dados de semiologia armada ou não, informações sobre patologias, evolução natural de afecções clínicas c das modificações do comportamento físico e psíquico gerados pela dor e imobilismo permitem que estratégias terapêuticas sejam instituídas visando, sempre que possível, a eliminação da condição nosológica ou fisiopatológica que acarretou a dor.

A participação em atividades de pesquisa favorece 0 ensino-aprendizagem pelo desenvolvimento do pensamento crítico, por estimular contato com a produção científica na área, por aproximar teoria e prática e por caracterizar situações de parceria entre professor e aluno. Podemos inferir que também na visão do aluno essa atividade é importante porque a participação em pesquisa, ou na elaboração de trabalhos científicos, foi uma categoria de resposta presente entre os motivos para participar da Liga e entre as fontes do sentimento de gratificação no trabalho na Liga. Dentre as insatisfações com as atividades na Liga de Dor relatadas pelos alunos, a não participação em projetos de pesquisa foi citada. Nos anos de 1995 e 1996 os alunos participaram de 12 projetos de pesquisa, que foram apresentados em eventos científicos nacionais e internacionais, organizaram 4 cursos sobre dor e 2 edições especiais da Revista de Medicina do Centro Acadêmico Osvaldo Cruz, versando sobre dor.

As opiniões dos alunos sobre suas atividades na Liga de Dor corroboram a idéia de que os estudantes são receptivos e interessados em aprender sobre esse tópico. Este é um dos fatores que contribuem para a promoção do ensino ${ }^{31}$ sobre dor o que é requisito essencial para que os profissionais estejam capacitados a prestar assistência o mais eficaz possível à pessoa que sente dor.

\section{CONCLUSÃO}

A Liga de Dor mostrou ser modelo adequado para desenvolver o ensino de aspectos relacionados ao tema dor junto a alunos de graduação de medicina e de enfermagem. As atividades assistenciais e de pesquisa foram veículos para esse processo. Os resultados são promissores. A maioria dos alunos expressou ter alcançado seus objetivos, total ou parcialmente, ter aumentado seu conhecimento sobre as síndromes álgicas e sobre o manejo do doente com dor, sentir-se gratificado com sua atividade, julgar que o tema dor deve ser incluído no currículo de modo mais enfático e que recomendaria a outros colegas a participação na Liga de Dor.

\section{REFERÊNCIAS BIBLIOGRÁFICAS}

1.BOND, M.R. Cancer pain: psychological substrates and therapy Int: FIELDS, H.L. et al. Advances in pain research and therapy: proceedings of the Fourth World Congress on Pain. New York, Raven, 1985. v.9. p.559-67

2.BONICA, J.J. Treatment of cancer pain: current status and future needs. In: FIELDS, H.L.et al. Advances in pain research and therapy: proceedings of the Fourth World Congress on Pain. New York, Raven, 1985. v.9, p.589-615.

3.BOULOS, M. Objetivos educacionais do ensino médico. Documentos CEDEM,São Paulo, FMUSP, 1994. p 45-4.

4.BUCHANAN, J. et al. Medical education in palliative care. Med. J. Aust.. v. 152, n.1, p. 27-9, 1990. 
5.CLEELAND, C. S. et al. Pain and its treatment in outpatients $w$ ith metastatic cancer, N. Engl. J. Med. v. 330, n. 9, p. 592-6. 1994

6.CLEELAND, C.S. Research in eancer pain- what we know and what we need to know. Cancer, v.67, 11.3. p.823-7, 1991. Supplement 3.

7.DINIZ, C. S. G. et al. Objetivos da escola médica. Ponto e Vírgula, Boletim FMUSP, CEDEM, n.33. p.8-11, 1995.

8.GRANT, M. G.; RIVERA L. M. Pain education for nurses, patients, and families. In: McGUIRRE. D.B.et al. Cancer pain management. 2ed. Boston, Jones and Bartley, 1995. Cap.11, p.289-319.

9.HIRAGA, K. et al. The incidence of cancer pain and improvement of pain management in Japan, Postgrad. Med. J., v.67, n.2, p.14-25, 1991. Supplement 2.

10.INTERNATIONAL ASSOCIATION FOR THE STUDY OF PAIN (IASP). Task Force on Professional Education. Core Curriculum for professional education in pain. 2ed. IASP Préss. Seattle, 1995.

11.JONES, W. L. et al. Cancer patient knowledge, beliefs, and behavior regarding pain control regimens: implications for education programs. Pat. Couns. Health Ed., v.5, n.4, p.159-64. 1984 .

12.KOSEKI, N. M.; BRUERA, E. Decisão médica ética em casos de pacientes terminais. Rev. Bras. Cancerol., v.42, n.1, p. $15-29,1996$

13.LEVIN, D. N et al. Public attitudes toward cancer pain. Cancer, v.56, n.9, p.2337.39, 1985.

14.MARCONDES, E. A. Monitorização do currículo de graduação médica. Ponto e Virgula, Boletim FMUSP, CEDEM, n.35, p.9-10, 1996.

15.MARISS; R. M.: SACHAR. E. J. Undertreatment of medical impatients with narcotic analgesics. Ann. Intern. Med., v.78, n.2, p.173-81, 1973.

16.MAX, M. B. Improving outcomes of analgesic treatment: is education enough? Ann. Int. Med., v.113, n.11, p.885-89, 1990.

17.MERSKEY. H. Pain and psychological medicine. In: WALL, P. D.: MELZACK R. Textbook of pain. Bed., Edinburgh, Churchill Livingstone, 1994. cap.47, p.903-20.

18.MORRIS, D. B. The culture of pain. Berkeley, University of Califormia Press, 1993.

19. NEWBLE, D. I. Continuing medical education. Med. J. Aust., v. $148, n .1, p .5-6.1988$.

20.NOBRE, M. R. C. et al. Qualidade de vida, educação em saúde e prevença de doenças. Qualimetria. v.6, n.32, 1994.

21. O'DONNELL J.F. BARON J.A. A strataegy to teach medical decision making within a medical school curriculum. J. Cancer Educ., v.6, 2. 3, p.123-28, 1991.

22. ORGANIZACION MUNDIAL DE LA SALUDE. Alívio del dolor en el cancer. Ginebra, 1987

23. PIMENTA. C. A. M. Aspectos afetivos, culturais e terapêuticos relacionados à dor no cáncer. São Paulo, 1995. Tese (Doutorado) - Escola de Enfermagem, Universidade de São Paulo.
24.PIMENTA, C. A. M.; KOIZUMI, M. S. Analgesia em câncer: crenças e atualizaçao. Rev. Esc. Enf. USP, v.27, n.2, p. $309-14,1993$.

25.PIMENTA, C. A. M. et al. Dor: ocorréncia e evoluçăo no pós. operatório de cirurgia cardíaca e abdominal. Rev. Paul. Enf., v.11, n.1, p. 3-10, 1992.

26.PORTER, J.; JICK, H. Addiction rare in patients treated with narcotics. N. Engl. J. Med., v.302, n. 2, p.123, 1980.

27.POSSO, M. N. S. et al. Pain therapeutics: an emergent discipline in nursing graduation. / Apresentado (Poster) ao Pain Management Into Nursing Education: An Update For Nurses Educators, Seatlle, 1996/.

28. RAVAUD, A et al. A survey in general practice about undergraduate cancer education: results from Gironge (France). J Cancer Edu., v.6, n.3, p.153-57, 1991.

29.ROENN, J. H. V. et al. Physician attitudes and practice in cancer pain management. Ann. Intern. Med., v.119, n.2, p.121-6, 1993 .

30.RYAN, P. et al. Cancer pain: knowledge, attitudes of pharmacologic management. J. Gerontol. Nurs., v.20, n.1, p. 7-16, 1994.

31.SEERS, K; DAVIS, P. Pain in the nursing curriculum. In: CARROL, D; BOWSHER, D. Pain management and nursing care. Oxford, Butterworth - Heinemann, , 1993. cap 4, p. 28-33.

32.TEIXEIRA, M. J. Dor. /Editorial/. Rev. Med., v.74, n.2 p.52-4, 1995.

33.TEIXEIRA, M. J. Tratamento neurocirúrugico da dor. In: RAIA A. A.; ZERBINI EJ (ed). Clínica cirúrgica Alípio Correa Netto, São Paulo, Sarvier, 1988. p. 541-72.

34.TEIXEIRA, M. J.et al. Dor: Conceitos Gerais, Sao Paulo, Limay, 1994.

35. TEIXEIRA, M. J. et al Dor no Brasil: estado atual e perspectivas. Sao Paulo, Limay, 1995.

36.WARD, N.G. Pain and depression In: BONICA, J. J. The management of pain, 2.ed. Philadelphia Lea \& Febeger, 1990. cap.18, p.310-19.

37.WHEDON, M.; FERRELL, B. R. Professional and ethical considerations in the use of high-tech pain management. Atlanta, American Cancer Society, Professional Education Publication, 1992.

38.WHITMAN, N. A. et al. A second look at consequences for medical education of problem solving in science and medicine. Acad Med, v.64, n.9, p.548-49, 1989.

39.WILKIE, D. The IASP Pain curriculum: what does it mean for nurses educators? In: OFFICIAL SATELLITE SYMPOSIUM TO THE WORLD CONGRESS ON PAIN, 8, Vancouver, 1996. Programs. Vancouver, 1996. p.1.

40.WILSON, J. F, et al. Medical students' attitudes toward pain before and after a brief course on pain. Pain, v.50, n.3, p.251-6, 1992. 